\title{
Correction haute densité par effet des microchamps électriques dans un plasma $Z$-pinch de deutérium
}

V. André, C. Fleurier et D. Hong

GREMI - CNRS, Université d'Orléans, 45067 Orléans cedex, France

\begin{abstract}
A Z-pinch plasma in Deuterium has been developped for heavy ion beam - plasma interaction measurements. The plasma diagnostic was achieved by means of a two wavelength laser absorption method and by emission spectroscopy. The diagnostic of the high density plasma was based on an iterative procedure coupling the two different measurements. It turned out that the only way to obtain a satisfying convergence of the procedure, in good agreement with the experimental results, was to use recent theories including the influence of internal electric microfields on the population densities in the plasma.
\end{abstract}

L'étude de I'interaction entre faisceaux d'ions lourds énergétiques et plasma a nécessité [1] le développement et le diagnostic d'une décharge de type Z-pinch. Le plasma est produit dans un tube de quartz ( $\varnothing 10.4 \mathrm{~cm}$, longueur $40 \mathrm{~cm}$ ), pour 2 millibars de deutérium. Un banc de condensateurs $(30 \mathrm{kV}, 10 \mu \mathrm{F})$ délivre la puissance électrique, conduisant à un maximum de courant de $190 \mathrm{kA}$ pour une inductance totale de $230 \mathrm{nH}$. Des images obtenues avec une caméra à balayage ont montré la stabilité du plasma pendant la compression, celle-ci étant maximum à 2.6us après l’ignition pour un diamètre de plasma de $2 \mathrm{~cm}$.

Le diagnostic du plasma a été réalisé par spectroscopie d'émission et par absorption laser. Les raies de Deutérium $D_{\alpha}$ et $D_{\beta}$ ainsi que le continu ont été enregistrés longitudinalement simultanément avec un OMA par le biais d'une fibre optique couplée à un spectromètre. Les raies étant larges, une faible résolution est requise. Le temps de pose est de 100ns. Par ailleurs, l'utilisation d’un laser Argon ionisé en mode multiraie a 
permis de mesurer simultanément le coefficient d'absorption longitudinal à 2 longueurs d'onde différentes.

Le diagnostic est délicat car le coefficient d'émission n'est composé que de rayonnement continu (absence de raies spectrales) au moment du maximum de compression. Les paramètres du plasma sont alors déterminés par une procédure itérative originale: (a) en fin de décharge, des approches théoriques des spectres expérimentaux utilisant les profils de raie théoriques [2] permettent de déterminer, outre les paramètres du plasma à ces instants, l'intensité absolue d'émission du plasma à tout instant; (b) à l'aide des lois de transfert radiatif, les mesures d'intensité couplées avec les mesures d'absorption donnent à tout instant l'intensité de corps noir, donc la température, du plasma; (c) connaissant l'intensité émise et la température, la densité électronique est alors aisément déduite. Malheureusement, lors de l'étape (b), il est impossible d'obtenir en fin de décharge une convergence acceptable de la température du plasma et en même temps des approches théoriques satisfaisantes pour les raies $D_{a}$ et $D_{\beta}$. Les erreurs expérimentales peuvent être écartées.

Par contre, des théories récentes [3] [4] montrent que les densités de population des différents niveaux d'énergie diminuent sous l'influence des microchamps électriques internes au plasma. Cette correction est d'autant plus importante que le niveau considéré est élevé. L'introduction de ces populations modifiées dans la procédure itérative conduit à des approches théoriques des spectres expérimentaux beaucoup plus satisfaisantes et à une bien meilleure convergence des paramètres du plasma. Au maximum de compression, on obtient ainsi une densité électronique de $1.3 \times 10^{18} \mathrm{~cm}^{-3}$ pour une température de $80000 \mathrm{~K}$.

En conclusion, ce diagnostic met en évidence expérimentalement les effets des microchamps électriques internes au plasma sur les densités de population à haute densité électronique. Des résultats plus complets sont disponibles en [5].

\section{Références:}

[1] M. Chabot, et al., Il Nuovo Cimento 106A 12 (1993), 1789

[2] H.R.Griem, Spectral Line Broadening by Plasma (Academic Press, NY, 1974)

[3] V. Sevastyanenko, Beitr. Plasmaphys. 25 (1985), 151

[4] D.G. Hummer D.Mihalas, Astrophys. J. 331 (1988), 794

[5] V.André, Thèse (Orléans, décembre 1993). 